\title{
Fratura orbitária provocada por projetil de borracha: Relato de caso
}

\author{
Orbitary fracture caused by rubber project: Case report \\ Fractura orbitaria causada por proyectil de caucho: Relato de caso
}

\author{
Mirlany Mendes Maciel Oliveira \\ ORCID: https://orcid.org/0000-0002-4744-8094 \\ Universidade Federal de Uberlândia, Brasil \\ E-mail: mirlany.mendess@gmail.com \\ Amanda Bárbara Borges \\ ORCID : https://orcid.org/0000-0001-7750-5494 \\ Universidade Federal de Uberlândia, Brasil \\ E-mail: amanda-borges19@hotmail.com \\ Rodrigo da Franca Acioly \\ ORCID: https://orcid.org 0000-0001-6535-9039 \\ Hospital Geral de Roraima, Brasil \\ E-mail: rodrigoaciolybmf@hotmail.com \\ Dennis Dinelly de Souza \\ ORCID: https://orcid.org/0000-0003-2711-0851 \\ Hospital Geral de Roraima, Brasil \\ E-mail: dennisouza@gmail.com \\ Daniel do Carmo Carvalho \\ ORCID: https://orcid.org/0000-0002-0368-8301 \\ Hospital Geral de Roraima, Brasil \\ E-mail: danielcarvalhobuco@hotmail.com \\ Bruno Araújo da Silva \\ ORCID: https://orcid.org/0000-0002-8493-5852 \\ Hospital Geral de Roraima, Brasil \\ E-mail: araujoodontologia@gmail.com
}

\begin{abstract}
Resumo
Os projeteis de borracha são utilizados, geralmente por entidades policiais e de segurança privada, na busca de um melhor controle de manifestações, tumultos ou rebeliões. Consideradas armas menos letais que as armas de fogo, tais artifícios podem causar lesões corporais devastadoras, com consequências estéticas, funcionais e sociais inimagináveis ao alvo. A utilização deste instrumento deve seguir recomendações criteriosamente na busca de evitar e/ou minimizar danos. As lesões balísticas que acometem a região maxilofacial consistem em um desafio para o cirurgião Bucomaxilofacial, em virtude do seu alto grau de complexidade, devendo ser realizado a identificação anatômica dos tecidos danificados, o entendimento das limitações desse tipo de lesão, a preservação de tecidos esqueléticos e moles. Desta maneira a conscientização do potencial lesivo deste instrumento deve ser prioridade na busca de minimizar lesões advindas do mesmo. O objetivo deste artigo é relatar o caso clínico de fratura orbitária causada por projétil de borracha, bem como discutir o potencial de morbidade deste instrumento.
\end{abstract}

Palavras-chave: Órbita; Fraturas orbitárias; Traumatismos faciais; Traumatismos cranianos penetrantes.

\begin{abstract}
Rubber projectiles are used, usually by police and private security entities, in the search for better control of demonstrations, riots or rebellions. Considered less lethal weapons than firearms, such devices can cause devastating bodily injuries, with aesthetic, functional and social consequences unimaginable to the target. The use of this instrument must follow recommendations carefully in order to avoid and / or minimize damage. The ballistic injuries that affect the maxillofacial region are a challenge for the buccomaxillofacial surgeon, due to its high degree of complexity, and anatomical identification of the damaged tissues, understanding of the limitations of this type of injury, the preservation of skeletal tissues and soft. Thus, awareness of the harmful potential of this instrument should be a priority in the quest to minimize injuries arising from it. The purpose of this article is to report the clinical case of an orbital fracture caused by a rubber projectile, as well as to discuss the morbidity potential of this instrument.
\end{abstract}

Keywords: Orbit; Orbital fractures; Facial injuries; Penetrating cranial injuries.

\section{Resumen}

Los proyectiles de goma son utilizados, generalmente por la policía y entidades de seguridad privada, en la búsqueda de un mejor control de manifestaciones, motines o rebeliones. Considerados armas menos letales que las armas de fuego, estos dispositivos pueden causar devastadores daños corporales, con consecuencias estéticas, funcionales y 
sociales inimaginables para el objetivo. El uso de este instrumento debe seguir cuidadosamente las recomendaciones para evitar y / o minimizar daños. Las lesiones balísticas que afectan la región maxilofacial son un desafío para el cirujano buccomaxilofacial, debido a su alto grado de complejidad, identificación anatómica de los tejidos dañados, comprensión de las limitaciones de este tipo de lesiones, preservación de tejidos esqueléticos y blandos. Por lo tanto, la conciencia del potencial nocivo de este instrumento debe ser una prioridad en la búsqueda de minimizar las lesiones derivadas de él. El propósito de este artículo es reportar el caso clínico de una fractura orbitaria causada por un proyectil de goma, así como discutir el potencial de morbilidad de este instrumento.

Palabras clave: Orbita; Fracturas orbitarias; Lesiones faciales; Lesiones craneales penetrantes.

\section{Introdução}

As armas de fogo são mundialmente utilizadas por diversos setores da sociedade, legalmente ou ilegalmente. Tais armas possuem um alto grau de letalidade e grande potencial de destruição, aumentando assim a complexidade em casos de fraturas faciais desencadeadas por estes instrumentos (Cruz, 2018; Fonseca et al., 2015).

$\mathrm{Na}$ busca de um maior controle por parte das autoridades policiais nos casos de manifestações, rebeliões ou tumultos, a utilização de armas não letais tem sido cada vez mais comuns, pois permitem neutralizar o oponente sem lhe causar graves danos. Entretanto, as armas não letais, como os projeteis de borracha, em determinados casos podem atingir regiões mais nobres do corpo desencadeando danos importantes e até fatais à vida humana (Mendes, 2018).

As balas de borrachas são projéteis menos letais que as munições convencionais das armas de fogo. Geralmente são constituídas de elastômero (polímero com propriedades elásticas) e podem ser encontradas em diversos calibres, tendo em sua cápsula um projétil cilíndrico ou vários esféricos. A cápsula que a envolve geralmente é constituída por propelente de pólvora e espoleta (Cruz, 2018). As dimensões desses projéteis podem variar de $3,5 \mathrm{~cm}$ a $9,15 \mathrm{~cm}$, pesar entre 135 a $140 \mathrm{~g}$ e ultrapassar a velocidade de $70 \mathrm{~m} / \mathrm{s}$ (Ifantides et al., 2020).

Existem mais de 75 tipos de balas de borracha no mundo e geralmente são comercializadas para militares e segurança privada. Esses projéteis atuam por meio da transferência de energia cinética. Quanto mais densos e maior velocidade imprimida, maior a força de impacto (Haar et al., 2017). A extensão de uma lesão causada por armas consideradas não letais vai depender de 7 elementos: a precisão da mira, o poder de ferimento do projétil, a distância do tiro, a presença de proteção, a área afetada, o estado fisiológico do indivíduo alvo e a rapidez e qualidade do pronto atendimento (Voiglio et al., 2004).

Em disparos com projéteis não letais deve-se manter uma distância mínima de 20 metros do instrumento ao alvo, tendo ainda como orientação o disparo direcionado para os membros inferiores, afim de minimizar os danos que os projeteis não letais podem causar (Cruz, 2018).

Quando essas recomendações não são seguidas, estruturas nobres como o globo ocular e a órbita estão ainda mais em risco, pois são delicados e com enorme potencial de serem lesionados. Embora não sejam consideradas letais, as balas de borracha podem causar a perda de visão, qualidade visual ou até mesmo do globo ocular, comprometendo a qualidade de vida e restringindo o indivíduo de inúmeras atividades (Ifantides et al., 2020).

As lesões balísticas que acometem a região maxilofacial consistem em um desafio para o cirurgião Buco-maxilofacial, em virtude do seu alto grau de complexidade. As lesões de tecidos moles, inseparáveis do trauma balístico, podem provocar avulsão, necrose e comprometimento da vascularização. Para reconstruções faciais por projeteis é fundamental a identificação anatômica dos tecidos danificados, o entendimento das limitações desse tipo de lesão, a preservação de tecidos esqueléticos e moles e a minimização de necessidade de reposição tecidual, sendo de suma importância a preservação da funcionalidade, forma e estética facial na busca de minimizar danos sociais e psicológicos (Fonseca et al., 2015).

A cavidade orbital é caracterizada como uma pirâmide óssea composta por 4 paredes: superior (teto), medial, lateral e inferior (assoalho). Sua principal função é a de sustentar e proteger o globo ocular. Por se tratar de uma estrutura frágil, 
fraturas da borda orbitária e de assoalho estão comumente associadas a fraturas do complexo zigomático-maxilar (Gart \& Gosain, 2014).

As fraturas orbitais podem ocorrer em 3 regiões anatômicas: na borda, na órbita central e no ápice. As fraturas isoladas de borda orbital são raras e geralmente acontecem em associação com outras fraturas do complexo zigomático-maxilar ou do tipo Lefort. Já as fraturas da órbita central, conhecidas como "blowout" são as mais comuns, tendo como os sítios mais acometidos o assoalho orbital e a parede medial, por terem pouco suporte estrutural. Por fim, as fraturas envolvendo o ápice orbital são ainda mais raras e geralmente associadas as fraturas de base de crânio (Strong, 2014).

Os principais sinais e sintomas de fraturas de assoalho orbital são: dor localizada, diplopia, equimoses periorbitais, edema palpebral, hemorragia subconjuntival e déficits sensoriais no nervo infraorbitário. Na presença desses indícios, deve ser realizado o exame físico por meio da inspeção da órbita, observando a presença de laceração ou degraus ósseos. Por fornecer informações relevantes sobre o tamanho e a posição da lesão, a Tomografia Computadorizada é o exame complementar de escolha, sendo os cortes coronal e axial com reconstruções tridimensionais os mais indicados para avaliação de fraturas do assoalho orbital (Gart \& Gosain, 2014).

A falta de dados sobre as indicações, o momento cirúrgico e a duração do acompanhamento no pós-operatório dificultam o gerenciamento das fraturas de assoalho orbital. O momento ideal de intervenção deve ser baseado nos sintomas do paciente e nos achados clínicos (Burnstine, 2002). O procedimento, os riscos e benefícios devem ser documentados e discutidos com clareza com o paciente. O tratamento das fraturas orbitais visa restaurar o volume orbital por meio da redução e fixação. Entretanto, na prática, esse processo é bastante complexo, prevalecendo o melhor resultado aquele não alterar o volume orbital (El- Hadadet al., 2021; Hartwing et al., 2019).

O presente artigo possui o objetivo relatar o caso de fratura orbital ocasionada por um artefato de borracha, bem como discutir a capacidade de morbidade deste instrumento.

\section{Metodologia}

O presente estudo é um relato de caso, que consiste em um tipo de estudo descritivo, retrospectivo, qualitativo, realizado por meio da técnica de observação direta. O paciente consentio na divulgação dos dados e exibição das imagens por meio da assinatura do Termo de Consentimento Livre e Esclarecido (Pereira et al., 2018).

\section{Relato de caso}

Paciente gênero masculino, 29 anos, melanoderma, foi conduzido por policias militares ao pronto atendimento, após ter sido atingido por projétil de borracha em região de face à direita. Após a avaliação inicial seguindo o protocolo A.T.L.S., foi encaminhado a equipe de Cirurgia e Traumatologia Buco-maxilo-facial. Ao exame extra-oral ficou evidente a presença de projétil de borracha em região infraorbitária esquerda associado a laceração tecidual, edema periorbital e epistaxe, com relato de dor em globo ocular ipsilateral. Na avaliação intra-oral não foram notadas alterações. Exame tomográfico revelou fratura em margem inferior e soalho de orbita e consequente herniação dos tecidos periorbitais para o seio maxilar (Figura 01 e 02). 
Figura 1 - Tomografia computadorizada pré-operatória. A- Reconstrução 3D; B- Corte sagital C- Corte coronal 1 D- Corte coronal 2.

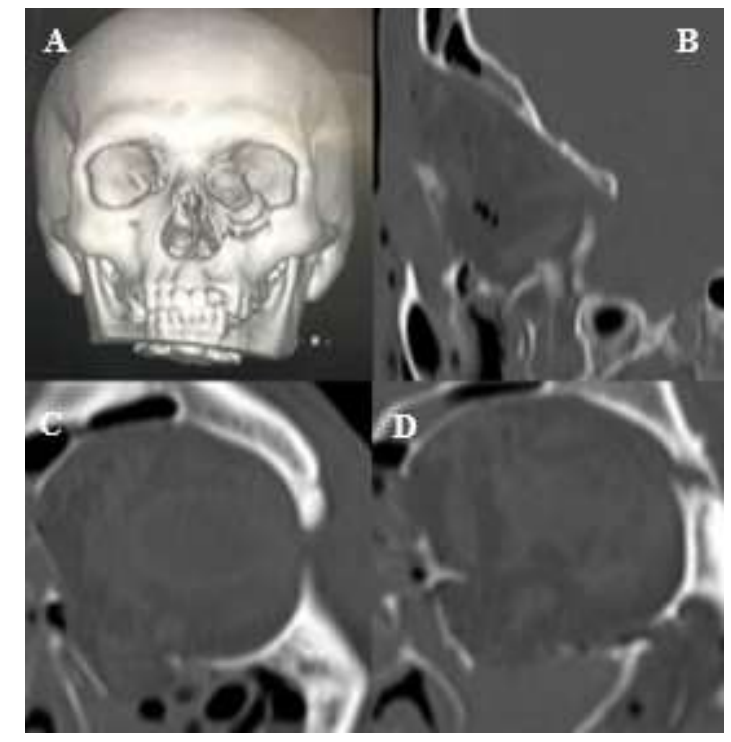

Fonte: Autores (2021).

Figura 2 - Remoção de projétil de borracha de face.

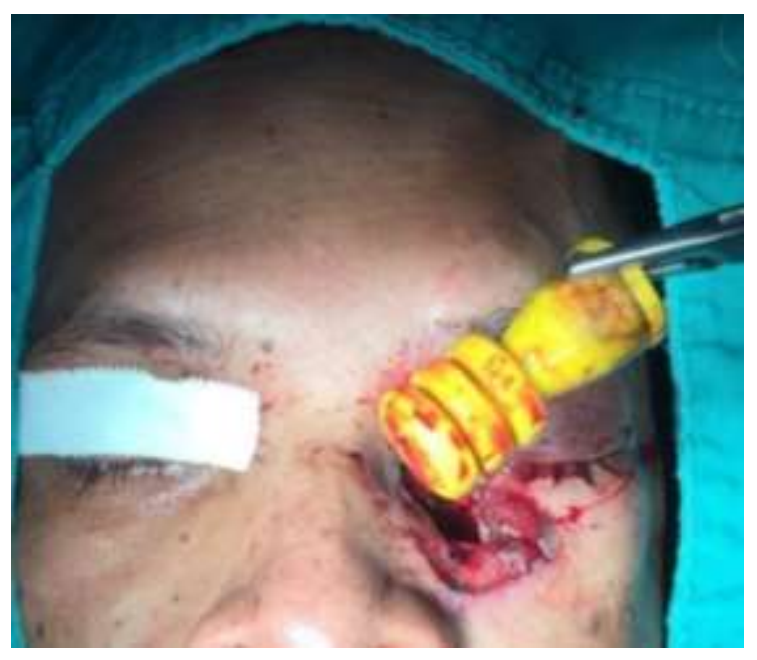

Fonte: Autores (2021).

O tratamento proposto foi redução e fixação da fratura orbitária sob anestesia geral. O paciente foi submetido a remoção cirúrgica do projétil, abundante lavagem utilizando soro fisiológico $0,9 \%$ associado a debridamento minucioso do tecido mole lesionado, e posteriormente reconstrução de fratura orbitária com tela de titânio, com a finalidade de restabelecer anatomicamente o assoalho orbital e dar sustentação aos tecidos orbitários. Após a fixação interna procedeu-se as suturas com Vicryl® 3-0 (poliglactina multifilamentado) em planos e nylon 5-0 (monofilamentado) em pele (Figuras 3). 
Figura 3 - Trans-operatório A- Exposição de fratura orbitária B- Reconstrução orbitária com tela de titânio.

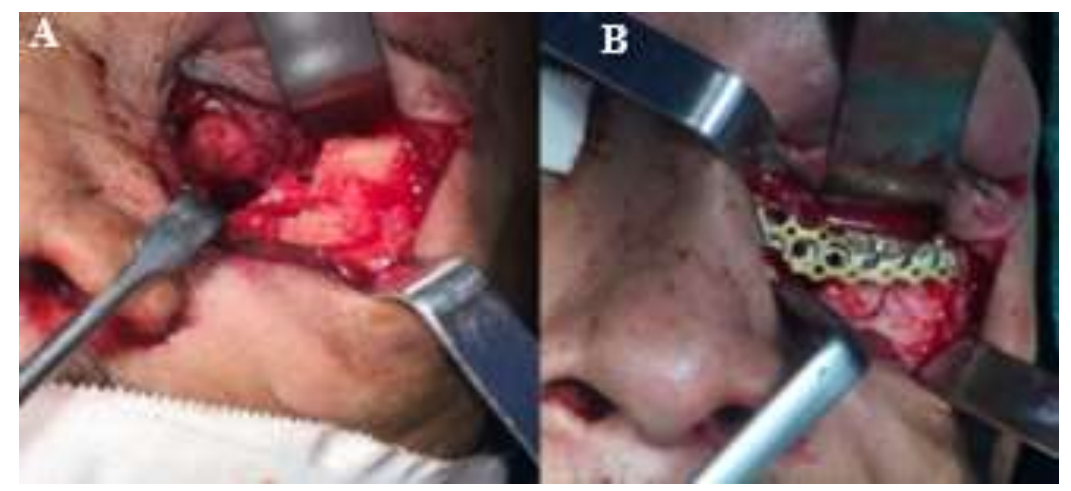

Fonte: Autores (2021).

Paciente realizou tomografia de face de controle que evidenciou tela de titânio em região de assoalho orbitário devolvendo contorno anatômico a cavidade orbitária (Figura 4).

Figura 4 - Tomografia computadorizada pós-operatória A- Corte sagital B- Corte coronal.

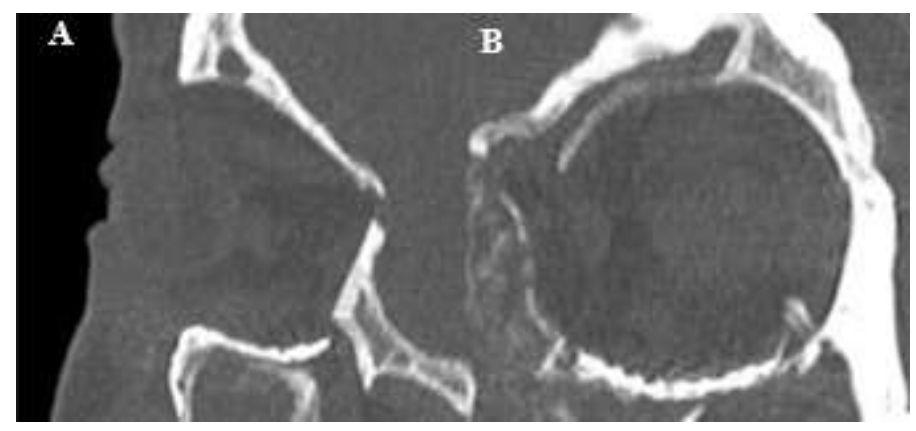

Fonte: Autores (2021).

Paciente foi acompanhado por um período de 45 dias de pós-operatório e não se observou complicações pósoperatórias com preservação de acuidade visual e ausência de enoftalmia ou diplopia.

\section{Discussão}

Atualmente existe uma grande variedade armas não letais, que incluem armas elétricas, irritantes químicos como spray de pimenta e munições de impacto. As balas de borracha se incluem no grupo de munições de impacto (Amelot et al., 2013).

O efeito esperado pelas autoridades que utilizam esse tipo de arma é o de incapacitação, mas não de morte ou dano permanente. Entretanto, o que se tem observado é que o resultado final pode ser uma deficiência permanente ou até mesmo a morte de um indivíduo. Dessa forma, o termo "arma de ferimento reduzido" é mais adequado que "arma não letal" (Voiglio et al., 2004).

A cavidade orbitária é particularmente sensível a fraturas por possuir ossos delgados e se encontrar em uma localização exposta (Gosau et al., 2011). As altas taxas de fratura de assoalho orbital por traumas podem ser explicadas devido a sua anatomia, que possui o maior grau de deformação em relação as outras paredes orbitais. O assoalho orbital é a parede 
mais fina da órbita, composta por 3 ossos: maxilar, zigomático e palatino. Ele é mais fino medialmente ao canal infraorbital, podendo medir 0,5mm de espessura (El-Hadad et al., 2021).

Em relação aos materiais utilizados para reconstrução do assoalho orbital fraturado, não há um consenso definitivo entre os cirurgiões. Geralmente, a decisão dependerá do tamanho do defeito e da preferência e experiência do cirurgião (Holtmannet al., 2016).

As telas de titânio possuem altas taxas de sucesso quando utilizadas para reconstruções orbitais complexas. Elas possuem as seguintes vantagens que incluem facilidade de manuseio, estabilidade, são radiopacas, possíveis de serem esterilizadas e estão prontamente disponíveis sem necessidade de sítio doador (Strong, 2014). Por esses motivos, diversos autores sugerem a reconstrução de fraturas de assoalho orbital com mais de 2,5-3,0cm com as telas de titânio (Holtmann et al., 2016; Gosau et al., 2011; El-Hadad et al., 2021), como foi realizado no caso em questão, o qual utilizou-se a tela de titânio para a reconstrução do assoalho orbitário.

O tipo de injúria causada por artefatos de borracha depende de vários fatores, tais como massa, o tamanho, a forma, velocidade do impacto, distância do disparo e o tipo de tecido que atinge. Em áreas com baixo limite elástico, como a face, são observadas com frequência severas penetrações (Amelot et al., 2013). Lavy e Asleh (2003) relatam em seu estudo clínico que 42 pacientes foram atingidos por balas de borrachas provocando lesões de variadas, apresentando as lacerações de pele, hifema e ruptura do globo as lesões oculares mais frequentes.

Um estudo retrospectivo sobre lesões oftálmicas causadas por armas não letais demonstrou que de 43 casos, 25 resultaram em fraturas orbitárias e rompimento de globo ocular (Ifantides et al., 2020). Outro estudo realizado no Chile, demonstrou que dentre 182 casos suspeitos de trauma ocular por balas de borracha 33 evoluíram com amaurose e 90 exibiram deficiência visual grave (Rodriguez et al., 2020).

No caso em questão, mesmo existindo acometimento das estruturas ósseas e significativa laceração facial o paciente não evoluiu com sequelas orbitárias, tais como amaurose e oftalmoplegia, condição esta que contraria os estudos de Khonsari et al. (2010), que relataram 2 casos de injurias maxilofaciais ocorridas por balas de borracha, evoluíram com a necessidade de evisceração do globo ocular.

Para de Lavy e Asleh (2003) as graves lesões oculares sugerem não haver o uso de uma distância segura para disparo contra o alvo e mesmo que em distâncias ditas corretas não se deve alvejar na direção da face em virtude dos grandes danos que estruturas nobres podem sofrer.

Ainda é possível verificarmos na literatura internacional relatos de ferimentos graves e até óbitos ocorridos em virtudes de ferimentos ocorridos por projéteis de borracha (Khonsari et al., 2010; Kobayashi et al., 2009).

As recomendações para utilização de balas de borracha em todo o mundo são similares e objetivam a contensão do alvo causando mínimas lesões. Variando a distância da arma ao alvo que segundo o estudo de Lavy \& Asleh (2003) seria de 40 metros porém, no Brasil há orientações de 20 metros (Cruz, 2018), o que são medidas muito divergentes elevando seriamente o risco de dano a vítima. Além de seguir rigorosamente critérios de disparo em direção aos membros inferiores.

A reconstrução orbital é uma tarefa bastante desafiadora pois resultados a quem do esperado podem desencadear morbidade, implicações estéticas, funcionais e psicológicas. O tratamento de traumas faciais ocasionadas por projéteis de borracha deve ser conduzido de maneira similar a projeteis de arma de fogo convencionais, com debridamento meticuloso, redução aberta de fraturas, fixação interna rígida evitando a ocorrência de infecções e de procedimentos secundários (Amaral et al., 2017).

As complicações mais comuns associadas à reparo cirúrgico de fraturas do assoalho orbital são: diplopia, enoftalmia e disfunções do nervo infraorbitário. A incidência de diplopia pós-operatória pode variar de 20 a 52\%, de disestesia pode chegar até $55 \%$ e enoftalmia até $27,5 \%$. Sendo que esses riscos são diminuídos quando o reparo é imediato (Gart \& Gosain, 2014). 


\section{Conclusão}

As balas de borracha são armas do tipo não letais, mas que podem ser prejudiciais, podendo desencadear a incapacitação, dano permanente ou até mesmo morte à depender da sua forma de utilização.

$\mathrm{O}$ treinamento dos profissionais e a disciplina quanto ao manejo das lesões causadas por projeteis são fundamentais para minimização dos danos ao alvo e melhor resultado final, visto os possíveis danos irrecuperáveis causados por balas de borracha. Portanto, fatores como treinamento adequado, incluindo treinamento tático e treinamento psicológico, são imprescindíveis para a condução efetiva de situações que necessitem da utilização de balas de borracha buscando minimizar a ocorrência de lesões grave ao alvo.

Em virtude da escassa quantidade de estudos referentes aos danos na face da utilização de balas de borracha, faz-se necessário o desenvolvimento de mais estudos relacionados ao manejo inicial, tratamento cirúrgico e a proservação desses casos na busca de minimização de morbidade e mortalidade de lesões provocadas por projétil de borracha.

\section{Referências}

Amaral, M. B. F., Bueno, S. C., Abdala, I. B. et al. (2017). Fraturas faciais causadas por armas de bala de borracha menos letais: relatório da série de casos e revisão da literatura. J Craniomaxillofac Surg. 21: 357- 361.

Amelot, A., Goutagny, S., Ricome, S. \& Peyre, M. (2013). Penetratring crânio cerebral injury cause by a rubber bullet question there lative harm lessness of the weapons. Am J Emerg Med. 31: 636e5-636e7.

Burnstine, M. A. (2002). Clinical recommendations for repair of isolated orbital floor fractures: An evidence-based analysis. Ophthalmology. 109(7):12071210 .

Colombo, L. R. C., Calderony, D. R., Rosim, E. T. \& Passari, L. A. (2011). Biomateriais para reconstrução da órbita: Revisão de literatura. Rev.Bras.Cir.Plast. 26(2):337-344.

Cruz, C. C. Chumbo fino. O Estado de São Paulo. http://www.estadao.com.br/noticias/geral,chumbo-fino, 843582.

El-Hadad, C., Deschenes J. \&mArthurs, B. (2021). Orbital floor fracture. Cmaj. 193(8):289.

Fonseca, R. J., Barber, H. D., Walker, R. V., Powers, M. P. \& Frost, D. E. (2015).Trauma Bucomaxilofacial. (4a ed.), Elsevier.

Gart, M. S., \& Gosain, A. K. (2014). Evidence-based medicine: Orbital floor fractures. Plastic and Reconstructive Surgery. 134(6):1345e-1355e.

Gosau, M., Schoneich, M. Draenert, F. G., et al. (2011). Retrospective analysis of orbital floor fractures-complications, outcome, and review of literature. Clinical Oral Investigations. 15(3):305-313.

Haar, R. J., Iacopino, V., Ranadive, N., et al. (2017). Death, injury and disability from kinetic impact projectiles in crowd-control settings: A systematic review. BMJ Open. 7(12):1-10.

Hartwig, S.,Nissen, M. C., Voss, J. O., et al. (2019). Clinical outcome after orbital floo,r fracture reduction with special regard to patient's satisfaction. Chinese Journal of Traumatology - English Edition. 22(3):155-160. https://doi.org/10.1016/j.cjtee.2019.01.002.

Holtmann, H., Eren H., Sander K., et al. (2016). Orbital floor fractures - short- and intermediate-term complications depending on treatment procedures. Head and Face Medicine. 12(1):4-9. http://dx.doi.org/10.1186/s13005-015-0096-3.

Ifantides, C., Deitzd, G. A., Christopher, K. L., et al. (2020). Less-Lethal Weapons Resulting in Ophthalmic Injuries: A Review and Recent Example of Eye Trauma. Ophthalmology and Therapy. 9(3) https://doi.org/10.1007/s40123-020-00271-9>.

Khonsari, R. H., Fleuridas, G., Arzul, L., Lefevre, F., Vincent, C. \& Bertolus, C. (2010). Severe facial rubber bullet injuries: Less lethal but extremely harm ful weapons. Injury. 41: 73-76.

Kobayashi, M. \& Mellen, P. F. (2009). Rubber Bullet Injury: Case Report with Autopsy Observation and Literature Review. Am J Forensic Med Pathol. $30(3): 262-267$

Lavy, T., \&Asleh, A. S. (2003). Ocular rubber bullet injuries. Nature, 17:821-824.

Mendes, P. S. P. Bala de Borracha. Repositório Institucional UFSC 2015. https://repositorio.ufsc.br/bitstream/handle/123456789/134575/baladeborra cha.comautor.pdf?sequence $=1$.

Pereira, A. S., Shitsuka, D. M., Parreira, F. J., \& Shitsuka, R. (2018). Metodologia em pesquisa científica. UFSM.

Rodriguez, A., Pena, S., Cavieres, I., et al. (2020) Ocular trauma by kinetic impact projectiles during civil unrest in Chile. Eye (Basingstoke). <http://dx.doi.org/10.1038/s41433-020-01146-w 
Research, Society and Development, v. 10, n. 6, e44710615976, 2021

(CC BY 4.0) | ISSN 2525-3409 | DOI: http://dx.doi.org/10.33448/rsd-v10i6.15976

Strong, E. B. (2014). Orbital fractures: Pathophysiology and implant materials for orbital reconstruction. Facial Plastic Surgery. 30(5):509-517.

Voiglio, E. J., Frattini, B. \& Dorrzapf, J. J. et al. (2004). Ballistic Study of the SAPL GC27 Gun: Is It Really“Nonlethal”? World J Surg. 28(4):402-405. 